\title{
BMJ Open Combined exercise and transcranial direct current stimulation intervention for knee osteoarthritis: protocol for a pilot randomised controlled trial
}

\author{
Wei-Ju Chang, ${ }^{1}$ Kim L Bennell, ${ }^{2}$ Paul W Hodges, ${ }^{3}$ Rana S Hinman, ${ }^{2}$ \\ Matthew B Liston, ${ }^{1}$ Siobhan M Schabrun ${ }^{1}$
}

To cite: Chang W-J, Bennell KL, Hodges PW, et al. Combined exercise and transcranial direct current stimulation intervention for knee osteoarthritis: protocol for a pilot randomised controlled trial. BMJ Open 2015;5:e008482.

doi:10.1136/bmjopen-2015008482

- Prepublication history for this paper is available online. To view these files please visit the journal online (http://dx.doi.org/10.1136/ bmjopen-2015-008482).

Received 15 April 2015 Revised 18 June 2015 Accepted 17 July 2015

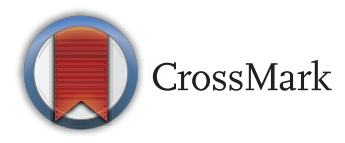

${ }^{1}$ University of Western Sydney, School of Science and Health, Penrith, New South Wales, Australia ${ }^{2}$ The University of Melbourne, School of Health Sciences, Parkville, Victoria, Australia ${ }^{3}$ The University of Queensland, School of Health and Rehabilitation Sciences, St Lucia, Queensland, Australia

Correspondence to Dr Siobhan M Schabrun; s.schabrun@uws.edu.au

\section{ABSTRACT}

Introduction: Osteoarthritis $(\mathrm{OA})$ is a major health problem and a leading cause of disability. The knee joint is commonly affected, resulting in pain and physical dysfunction. Exercise is considered the cornerstone of conservative management, yet metaanalyses indicate, at best, moderate effect sizes. Treatments that bolster the effects of exercise, such as transcranial direct current stimulation (tDCS), may improve outcomes in knee OA. The aims of this pilot study are to (1) determine the feasibility, safety and perceived patient response to a combined tDCS and exercise intervention in knee $\mathrm{OA}$, and (2) provide data to support a sample size calculation for a fully-powered trial should trends of effectiveness be present.

Methods and analysis: A pilot randomised, assessor-blind and participant-blind, sham-controlled trial. 20 individuals with knee $0 \mathrm{~A}$ who report a pain score of 40 or more on a $100 \mathrm{~mm}$ visual analogue scale on walking, and meet a priori selection criteria will be randomly allocated to receive either: (1) active tDCS plus exercise, or (2) sham tDCS plus exercise. All participants will receive 20 min of either active or sham tDCS immediately prior to 30 min of supervised muscle strengthening exercise twice a week for 8 weeks.

Participants in both groups will also complete unsupervised home exercises twice per week. Outcome measures of feasibility, safety, pain, disability and pain system function will be assessed immediately before and after the 8-week intervention. Analyses of feasibility and safety will be performed using descriptive statistics. Statistical analyses will be used to determine trends of effectiveness and will be based on intention-to-treat as well as per protocol.

Ethics and dissemination: This study was approved by the institutional ethics committee (H10184). Written informed consent will be obtained from all participants. The results of this study will be submitted for peerreviewed publication.

Trial registration number: ANZCTR365331.

\section{INTRODUCTION}

Osteoarthritis (OA) is a major public health problem and a leading cause of disability.

\section{Strengths and limitations of this study}

- A randomised, assessor-blind and participantblind, sham-controlled trial.

- Our study is the first to provide information on the feasibility and safety of a combined brain stimulation and exercise intervention in knee osteoarthritis.

- If trends of effectiveness are present, may provide data for a fully powered trial.

- This is a feasibility study and as such, is not powered to determine treatment effectiveness.

- The treating physiotherapist is not blinded to group allocation.

The knee joint is commonly affected and it is estimated that $10 \%$ of people aged over 60 years experience knee OA symptoms, ${ }^{1}$ resulting in substantial pain and physical dysfunction. ${ }^{2}{ }^{3}$ Current evidence demonstrates beneficial effects of exercise therapy on pain and physical function in knee OA, without the common and sometimes serious side effects associated with pharmacological and surgical interventions. ${ }^{4}$ Consequentially, exercise is considered the cornerstone of conservative management and is recommended in all clinical guidelines internationally. ${ }^{5} \quad 6$ Although exercise is effective in knee OA, meta-analyses indicate its treatment benefits are moderate for pain (standardised mean difference (SMD) $-0.49,95 \%$ CI -0.39 to -0.59 ) and physical function (SMD -0.52 , $95 \%$ CI -0.39 to -0.64$),{ }^{7}$ and are similar to those achieved with pharmacological treatments. ${ }^{8}$ Novel treatments that bolster the effect of exercise therapy have the potential to further improve outcomes in knee OA.

$\mathrm{OA}$ is a joint disorder that affects the cartilage and bone. Although pain is often attributed to localised joint pathology, research has shown that pain intensity does not always 
correlate with the severity of joint structural damage or the presence of inflammation. ${ }^{910}$ This discrepancy may be explained by the presence of a central component to persistent pain in knee OA. For example, nociceptive input from structural joint changes in OA may enhance the excitability and efficiency of synapses in the central pain pathway, a phenomenon termed central sensitisation. ${ }^{11}$ In support of this hypothesis, a recent systematic review demonstrated that central sensitisation contributes to persistent OA pain, characterised by local and widespread hyperalgesia (such as reduced pressure and thermal pain thresholds), increased spinal excitability (increased nociceptive flexion withdraw reflex), and deficits in descending pain processing (altered conditioned pain modulation) ${ }^{12}$ Treatments that target central sensitisation and pain processing may, therefore, be effective in knee OA.

The safe and painless application of weak direct electrical currents over the scalp (transcranial direct current stimulation, tDCS) is a novel intervention that has the potential to reduce central sensitisation and improve pain processing in knee OA. Using surface electrodes, direct current applied to the brain can increase (anodal stimulation) or decrease (cathodal stimulation) excitability of the region below the electrode as well as distant interconnected areas. ${ }^{13-15}$ Studies of healthy individuals and patients with persistent pain suggest anodal tDCS applied to the primary motor cortex can reduce pain, a finding thought to be explained by direct effects of stimulation on the cortex and thalamus, ${ }^{16-21}$ as well as 'downstream' effects on the anterior cingulate cortex and upper brain stem. ${ }^{22}{ }^{23}$ However, there has been no research investigating the effect of tDCS, whether applied alone or in combination with other interventions, in people with osteoarthritic pain. Exercise, moreover, can exert central as well as peripheral effects. Exercise treatments can alter sensory input from the periphery by modification of muscle control (ie, muscle coordination and strength) and through improved proprioception to enhance control of the affected joint, thus reducing nociceptor discharge and enhancing normal sensory input. Centrally, exercise is known to have an analgesic effect that reduces pain sensitivity in healthy individuals. ${ }^{24}{ }^{25}$ This is thought to be due to activation of opioidergic mechanisms and enhanced descending pain control systems. ${ }^{26} 27$ Treatments that modify peripheral inputs (exercise), and treatments that modify processing of these inputs at the supraspinal level (tDCS and exercise) may summate to produce greater effects on pain and function. In addition, tDCS has the potential to increase the brain's receptiveness to other interventions by increasing cortical excitability, a phenomenon known as priming. ${ }^{28}$ Thus, tDCS may optimise the responsiveness of the brain to exercise and improve outcomes beyond that which can be achieved with tDCS or exercise alone. Despite this, no study has examined the effect of a combined tDCS and exercise intervention in any persistent pain condition.
Therefore, the aims of this pilot randomised controlled trial are to (1) determine the feasibility, safety and perceived patient response to a combined tDCS and exercise intervention in knee OA and (2) provide data to support a sample size calculation for a fully-powered trial should trends of effectiveness be present.

\section{METHODS AND ANALYSIS \\ Trial design}

We will conduct a pilot randomised, assessor-blind and participant-blind, controlled trial. The trial will be reported according to the Consolidated Standards of Reporting Trials (CONSORT) statement for non-pharmacological treatment, ${ }^{29}$ and the template for intervention description and replication (TIDieR) checklist and guide.$^{30}$ It has been prospectively registered with the Australian and New Zealand Clinical Trials Registry (365331).

\section{Participants}

Participants with knee OA that meet the American College of Rheumatology clinical classification for idiopathic knee OA criteria ${ }^{31}$ will be recruited from the community. The criteria include the presence of knee pain plus at least three of the following six items: age over 50 years, morning stiffness lasting less than $30 \mathrm{~min}$, crepitus, bony tenderness, bony enlargement and no palpable warmth. A minimum pain score of 40 on a $100 \mathrm{~mm}$ visual analogue scale (VAS) on walking in the last week will be required. The main exclusion criteria are: (1) knee surgery in the past 6 months; (2) knee joint replacement or high tibial osteotomy on the affected side; (3) other muscular, joint or neurological conditions affecting lower limb function; (4) unable to walk unaided; (5) currently undertaking a structured exercise programme for knee OA or (6) contraindications to tDCS (eg, epilepsy) or conditioned pain modulation techniques (eg, loss of sensation). Participants can continue to use their normal medication for the duration of the trial. The type of medication and dosage used will be recorded at the baseline assessment.

\section{Recruitment}

Participants will be recruited from local arthritis support groups, social media and healthcare providers (medical practitioners, rheumatologists and physiotherapists). Potential participants will first complete an online screening questionnaire to determine their eligibility. Those who meet the inclusion criteria will be contacted by one of the investigators to confirm their willingness to participate in the trial and to arrange the baseline assessment of outcomes. Participants will provide written informed consent on arrival for baseline assessment.

\section{Randomisation}

Participants will be individually randomised on a 1:1 basis to the active or control groups in equal numbers. The randomisation schedule will be concealed in 
consecutively numbered, sealed opaque envelopes. An investigator not involved in recruitment or assessment will provide the envelope to the treating clinician who will reveal group allocation.

\section{Blinding}

Participants and the outcome assessor will be blinded to group allocation. The treating physiotherapist will deliver the tDCS intervention and the exercise therapy and will, therefore, not be blinded to group allocation. The success of participant blinding will be assessed at the follow-up assessment using a 'yes/no' response to the question "Do you feel you received the real brain stimulation?" and a $10 \mathrm{~cm}$ VAS of the individual's confidence in that judgement. Participants will also be asked "Why do you believe you received the real/sham brain stimulation?" and "Was it divulged to you whether you were receiving real brain stimulation or not?". The success of assessor blinding will be determined at the completion of the follow-up assessment for each participant using a 'yes/no' response to the question "Did you know which intervention group the participant was assigned to before completion of the follow-up assessment?" and "If you answer 'yes', how was it divulged to you?".

\section{Intervention}

Participants will be randomly allocated to one of two treatment groups: (1) active tDCS plus exercise or (2) sham tDCS plus exercise. All participants will receive 20 min of either active or sham tDCS immediately prior to $30 \mathrm{~min}$ of supervised muscle strengthening exercise, two times per week for 8 weeks. A qualified physiotherapist who is trained in the use of tDCS will deliver the tDCS interventions and the exercise therapy in a consulting room of the UniClinic at the University of Western Sydney. The physiotherapist has a Bachelor of Science in Physiotherapy and 6 years of clinical experience. Participants in both groups will also be instructed to complete home exercises twice per week to mimic typical clinical practice. Outcome measures will be assessed immediately before and immediately after the 8-week intervention.

\section{Transcranial direct current stimulation}

Participants will be comfortably seated in an armchair while receiving tDCS and will be asked to remain quiet for the duration of the intervention. tDCS will be delivered for $20 \mathrm{~min}$ using a direct current stimulator (DC-STIMULATOR, neuroConn, Ilmenau, Germany) via two $35 \mathrm{~cm}^{2}$ surface sponge electrodes. The active electrode (anode) will be placed over the primary motor cortex contralateral to the side of worst pain and the reference electrode (cathode) over the contralateral supraorbital region. ${ }^{21}$ Current intensity will be ramped up $(0-1 \mathrm{~mA})$ and down $(1-0 \mathrm{~mA})$ over $10 \mathrm{~s}$ at the beginning and end of the stimulation period. For sham stimulation, electrodes will be placed in an identical position.
Stimulation will be turned on for $15 \mathrm{~s}$ and then off, to provide the initial itching sensation. Participants will be informed that they may or may not perceive any sensation during the treatment. This procedure has been shown to effectively blind participants to the stimulation condition. $^{32}$

\section{Exercise}

Immediately following the tDCS intervention, participants will start one-to-one exercise therapy supervised by the physiotherapist. A standardised set of quadriceps strengthening exercises that are known to be effective in knee OA (table 1) will be performed with ankle cuff weights or resistance bands where necessary. ${ }^{73}$

Exercise intensity will be progressed by the physiotherapist as appropriate for each participant. Each exercise session will last $30 \mathrm{~min}$. A home exercise plan will be developed, monitored and progressed by the physiotherapist for each participant. An exercise diary with written and visual instructions for each exercise (including dosage) will be provided to each participant. The exercise diary will include space for participants to outline which exercises were completed, how many repetitions were performed and any comments regarding the home exercise programme (eg, whether pain was present, whether any exercises were difficult and if applicable, the reason why exercises were unable to be completed). The exercise diary will be returned to the investigator at the follow-up assessment session.

As the aim of this trial is to use tDCS to boost the effect of exercise, the intervention duration has been selected based on the number of sessions required to achieve efficacious outcomes using exercise alone in knee OA. An 8-week exercise duration period has been chosen based on evidence that at least 12 sessions of supervised exercise are required for exercise to be effective in knee $\mathrm{OA},{ }^{7}$ with a number of studies demonstrating symptom improvement in knee OA after 8-12 weeks of exercise. $^{34}$ Thus, 8 weeks (16 sessions) should be sufficient to show improvement in this population.

\section{Outcome measurements}

Baseline and follow-up assessments will be performed within 1 week of the participant starting or completing the intervention, respectively. All outcome measures will be performed in the research laboratories of the University of Western Sydney.

\section{Measures of pain and function}

Knee pain and function will be measured using (1) a $100 \mathrm{~mm}$ VAS with pain on walking over the last week selfassessed with terminal descriptors of 'no pain' (score $0 \mathrm{~mm}$ ) and 'extreme pain' (score $100 \mathrm{~mm}$ ). (2) The Western Ontario and McMaster Universities (WOMAC) OA index (24 items, total score $=96$ ) (Likert V.3.1) and its pain subscale ( 7 items, total score $=28)$ and physical function subscale ( 17 items, total score $=68$ ). This is a disease-specific self-report instrument that has been 
Table 1 Description of the strengthening exercise programme with images, progression and repetitions
Exercise Description
1. Knee extensor strengthening
- Seated knee extensions with ankle weights
- In a seated position, slowly straighten symptomatic knee until it is fully straight
- Hold for $5 \mathrm{~s}$ and then lower slowly
2. Hip abductor strengthening
Level 1:
- Side lying hip abduction with ankle weights
- Keep body still and knee straight, and lift affected leg up
- Do not swing affected leg forward
- Keep heel of foot higher than toes and behind hips while lifting leg straight upwards towards the ceiling
- Hold for $5 \mathrm{~s}$ and then lower slowly
Level 2:
- Standing hip abduction with thera-band elastic resistance band
- Place looped thera-band elastic resistance band around both legs just above the ankle
- Adequate tension on the elastic band and correct upright posture, with shoulders and hips both facing forward, is required prior to starting the exercise
- The back of a chair or a wall can be used to provide support
- Hold for $5 \mathrm{~s}$ and then lower slowly

3. Weight-bearing knee/hip extensor strengthening Level 1:

- Partial wall squats (option shown is to add thera-band elastic band around knees to incorporate the hip abductor muscles)

- Stand with one foot $30 \mathrm{~cm}$ away from the wall with feet apart and turned inwards

- With back straight and trunk and buttocks against a wall, slowly slide down the wall (as if to sit) to approximately $60^{\circ}$ (less if painful) and then back up again while keeping contact with the wall at all times

- Knees must go past the toes during the squat exercise

- Hold position for $5 \mathrm{~s}$

Level 2:

- Sit-to-stand (option to add thera-band elastic band around knees to incorporate hip abductor muscles)

- Seated with back against a chair of standard height with firm seat, slowly stand up without using hands for support

- Lean forward over toes so that the buttocks are lifted and hips go under the trunk

- Hold for $3 \mathrm{~s}$ with buttocks slightly off the chair before sitting back down slowly

Level 3:

- Alternate split sit-to-stand

- Place the foot of the unaffected leg $10 \mathrm{~cm}$ in front of the other foot

$\begin{array}{ll}\text { Progression } & \text { Repetitions } \\ \text { Ankle weights } & 3 \text { sets of } 10 \\ & 30 \text { s break period in } \\ & \text { between sets }\end{array}$

Increase ankle weights or progress to level 2

3 sets of 10 $30 \mathrm{~s}$ break period in between sets

Increase thera-band elastic band resistance

3 sets of 10

$30 \mathrm{~s}$ break period in between sets

3 sets of 10 $30 \mathrm{~s}$ break period in between sets

3 sets of 10 30 s break period in between sets increase elastic band resistance strength Progress further to level 3

Increase depth of squat

3 sets of 10 $30 \mathrm{~s}$ break period in between sets 
Table 1 Continued

\section{Exercise Description}

Progression

Repetitions

- Slowly stand by leaning forward with back straight (nose in front of the toes) and squeeze buttock muscles. Most weight bearing must be on the symptomatic knee

- Hold for $3 \mathrm{~s}$ with buttocks slightly off the chair before sitting back down, slowly

Level 3+:

- Split partial wall squats

- Slowly slide down the wall (as if to sit) keeping the trunk and buttocks in contact with the wall. Knees must move over the toes. Most weight bearing must be on the symptomatic knee

- Stop when symptomatic knee is bent to approximately $60^{\circ}$ (less if painful)

- Hold for $5 \mathrm{~s}$ and then slowly slide back up keeping the trunk and buttocks in contact with the wall at all times

\section{Hamstring strengthening seated knee} extensions

- Place a looped thera-band elastic resistance band around the leg of a heavy table or chair

- Seated in a chair, place the symptomatic leg in the looped thera-band elastic band with the knee slightly bent

- Slowly pull the leg backwards into the elastic band until the knee is bent and a strong resistance is felt

- Hold for $5 \mathrm{~s}$

5. Steps

(A). Step ups:

- Place symptomatic leg onto the step

- Slowly step up onto the step

- Touch foot of non-affected leg onto the step then place both feet back onto the starting position on the ground

(B). Step downs:

- Start with both legs standing on top of the step

- Bend the knee of the affected leg slowly to lower the non-affected leg towards the ground

Increase depth of squat

Increase elastic band resistance

First increase the height of the step and second, add weight

Weight can be held across the chest with both hands or use two hand weights

First increase the height of the step and second, add weight

Weight can be held across the chest with both hands or use two hand weights
3 sets of 10 $30 \mathrm{~s}$ break period in between sets

3 sets of $1030 \mathrm{~s}$ break period in between sets

3 sets of 10 30-60 s break period in between sets

\section{3 sets of 10} $30-60$ s break period in between sets

- Then straighten the affected knee slowly to return to the starting position

- The knee of the affected leg must point forward during the movement

Progression through the levels is an important component of the programme.

shown to be valid, reliable and responsive in an extensive range of studies of people with OA. (3) Global perceived effect of treatment, where each participant's perceived response to therapy is assessed using a sevenpoint Likert scale ranging from 'completely recovered' to 'vastly worsened'. This outcome will only be used in the follow-up assessment.

\section{Measures of pain mechanisms}

Measures of pain mechanisms will be performed in the same order for all participants.
I. Pressure pain thresholds (PPT): PPT will be measured using a hand-held pressure algometer (FORCE TEN FDX compact digital force gauge, Wagner Instruments, USA). The probe $\left(\right.$ size $\left.1 \mathrm{~cm}^{2}\right)$ will be applied perpendicular to the skin (rate $40 \mathrm{kPa} / \mathrm{s}$ ) until the participant first reports that the sensation of pressure has changed to pain. PPTs will be measured three times at each of the following sites: (1) ipsilateral tibialis anterior $(10 \mathrm{~cm}$ distal to the tibial tuberosity) and (2) ipsilateral extensor carpi radialis longus $(10 \mathrm{~cm}$ distal to the lateral epicondyle of the humerus) and eight sites in the peripatellar region: 
(1) $2 \mathrm{~cm}$ distal to the inferior medial edge of patella;

(2) $2 \mathrm{~cm}$ distal to the interior lateral edge of patella;

(3) $3 \mathrm{~cm}$ lateral to the midpoint of the lateral patellar

border; (4) $2 \mathrm{~cm}$ proximal to the superior lateral edge of patella; (5) $2 \mathrm{~cm}$ proximal to the midpoint of the superior patellar border; (6) $2 \mathrm{~cm}$ proximal to the superior medial edge of patellar; (7) $3 \mathrm{~cm}$ medial to the midpoint of the medial patellar border and (8) centre of the patella. ${ }^{35}$ The average of the three measurements at each site will be used in the analysis. PPT measures have been shown to be reliable in knee OA (intraclass correlation coefficient (ICC) $=0.83(0.72-0.90)) .^{36}$

II. Heat pain thresholds (HPT): HPT will be measured using the conditioned pain modulation system (Thermal Sensory Analyser, TSA-2001, Q-Sense-CPM, Medoc Ltd, Ramat Yishai, Israel). A $30 \times 30 \mathrm{~mm}$ Peltier-based thermode will be placed on the skin. The temperature will start at $32^{\circ} \mathrm{C}$ and increase at a rate of $0.5^{\circ} \mathrm{C} / \mathrm{s}$. Participants will push a button when the sensation of heat first turns to a sensation of pain. HPTs will be measured around the peripatellar region (3 sites: medial, patella and lateral knee joint lines) and at the bilateral ventral aspect of the forearm $(10 \mathrm{~cm}$ distal from the elbow crest). Three measurements will be recorded at each site and the average at each side will be analysed. HPT measure have been shown to have moderate reliability in knee OA (ICC=0.77 (0.62-0.87)) ${ }^{36}$

III. Conditioned pain modulation (CPM): CPM is a wellestablished, reliable and safe measure of pain processing that is thought to indicate the function of descending pain control systems. This is examined as a change in the pain perceived in one body region (test stimulation) as a result of pain induced in another body region (conditioned stimulation). We will use PPT measurement as the test stimulation and heat pain $\left(1^{\circ} \mathrm{C}\right.$ above HPT $)$ as the conditioned stimulation using the CPM System (Thermal Sensory Analyser, TSA-2001, Q-Sense-CPM, Medoc Ltd). Three PPTs (test stimulation) will be measured before the application of heat pain (conditioned stimulation). The heat pain will then be applied via a $30 \times 30 \mathrm{~mm}$ thermode. Three PPT measurements will be repeated $30 \mathrm{~s}$ after applying the conditioned stimulation. Participants will be asked to rate their pain during conditioned stimulation on a numeric rating scale $(0-100)$ at $0 \mathrm{~s}, 30 \mathrm{~s}$ and at the end of the trial. Pain scores will be maintained between 50/100 and 80/100 during testing. Participants will complete two trials in random order: (1) test stimulation at knee and conditioned stimulation at the contralateral forearm and (2) test stimulation at the contralateral forearm and conditioned stimulation at the ipsilateral forearm. The CPM paradigm has shown good intrasession reliability (ICC $>0.75) .{ }^{37}$

IV. Nociceptive flexor withdrawal reflex (NFR): NFR is a measure of central sensitisation and descending pain control systems, and was used in previous studies investigating central sensitisation in knee $\mathrm{OA}^{38}$ Surface stimulating electrodes will be positioned at a retromalleolar location along the sural nerve on the side of the painful knee. Recording electrodes will be positioned over the belly of the biceps femoris muscle. Stimulation will consist of five rectangular pulses of $1 \mathrm{~ms}$ duration with a $3 \mathrm{~ms}$ interval, and will proceed using an up and down staircase method. The intensity needed to evoke a response from the biceps femoris (indicating activation of the NFR), the area of the NFR response and the subjective pain threshold will be recorded. The NFR is a reliable experimental test (intersession coefficient of variation $\left(\mathrm{CV}_{\mathrm{SEM}}\right)=16.9 \%$, ICC $\left.=0.82\right){ }^{39}$

\section{Sample size and analysis}

This is a pilot study designed to generate data that can be used to inform a future large randomised controlled trial should the intervention appear feasible, safe and show trends of effectiveness. Thus, we have selected a sample size of 10 individuals per group, or a total of 20 participants. A sample size of 20 participants was selected as this is considered achievable within the time frame allocated for completion of the pilot study according to study recruitment rates within the laboratory. We aim to evaluate key trial parameters, such as recruitment and retention of participants, randomisation, levels of missing data and preliminary indications of effectiveness, to inform calculation of a sample size for powering a full trial. As this was a pilot study, a prospective sample size calculation was not conducted.

Data for feasibility and safety will be analysed using descriptive statistics. The percentage of participants who (1) meet the inclusion criteria, (2) agree to be randomised, (3) complete the intervention and (4) attend the follow-up assessment will be calculated. Feasibility will be measured as (1) the number of sessions attended by each participant, (2) number of drop-outs in each group, (3) proportion of participants recruited from the total number screened, (4) willingness of each participant to undergo therapy on an 11-point numerical rating scale with 'not at all willing' at 0 and 'very willing' at 10 (measured at baseline) and (5) the number of home exercise sessions completed. Safety will be presented as any adverse reaction reported on verbal questioning by the treating physiotherapist at each session. An adverse reaction is defined by WHO as "a response to a drug [intervention] which is noxious and unintended, and which occurs at doses normally used in man for the prophylaxis, diagnosis, or therapy of disease, or for the modification of physiological function" and that likely has a causal relationship with the intervention. $^{40} \mathrm{~A}$ mild tingling or itching sensation under the electrodes, fatigue, headache, nausea and insomnia have been reported as potential adverse reactions following tDCS. ${ }^{41}$ Potential adverse reactions as a result of the physical component of the intervention 
(muscle strengthening exercise) may include increased pain or muscle soreness around the knee joint and thigh. As potential adverse reactions are distinct for each component of the intervention, and as this trial includes a control group receiving sham tDCS, while both groups receive exercise, it should be possible to attribute any reported adverse reactions to either tDCS or exercise. The treating physiotherapist will record a description of any adverse reactions along with the severity, duration and how the adverse reaction was managed. The number of participants reporting adverse reactions, and the duration and severity of the adverse reactions will be reported.

To determine trends of effectiveness, analyses of pain, disability and pain system function will be performed according to intention-to-treat and per protocol using analysis of variance (ANOVA) to assess the changes within groups and the differences between groups over time (pre/post). Repeated measures of ANOVA will be used with factors of intervention (active tDCS/sham tDCS) and time (pre-intervention/post-intervention) as separate twolevel factors. Effect size will be determined using partial $\eta^{2}$ from planned contrasts. The size of the treatment effects will be used to determine whether it is worthwhile to conduct a full randomised controlled trial in the future. ${ }^{43}$ Given the pilot nature of this trial, missing data will not be replaced. Bonferroni post hoc tests will be applied if appropriate. The $\alpha$ will be set at 0.05 .

Means and standard deviations (SDs) for measures of pain, function and pain mechanisms will be used to perform a sample size estimate. Power will be set at $80 \%$ to detect between-group differences, with an $\alpha$ of 0.05 and a drop-out rate based on that of the pilot trial. SigmaPlot will be used to analyse the data in this trial.

\section{DISSEMINATION}

All participants will provide written informed consent following verbal and written explanation of the study protocol and the opportunity to ask questions. Participants are free to withdraw from the trial at any time without prejudice to future treatment. Results will be presented at scientific meetings and published in peer-reviewed journals. All publications and presentations related to the study will be authorised and reviewed by the study investigators.

\section{TRIAL STATUS}

This trial is currently recruiting and is expected to be completed (including follow-up testing) by August 2015.

\section{Twitter Follow Siobhan Schabrun at @DrSMSchabrun}

Contributors W-JC, MBL, KLB, RSH, PWH and SMS were involved in the conception, design, writing and editing of the study protocol. All the authors approved the final protocol.

Funding This work was supported by Arthritis Australia (The Zimmer Australia Grant). W-JC, PWH, KLB, MBL and SMS received salary support from the National Health and Medical Research Council of Australia, and RSH from the Australian Research Council.
Competing interests None declared.

Ethics approval The University of Western Sydney Human Research Ethics Committee has approved the trial (H10184).

Provenance and peer review Not commissioned; externally peer reviewed.

Open Access This is an Open Access article distributed in accordance with the Creative Commons Attribution Non Commercial (CC BY-NC 4.0) license, which permits others to distribute, remix, adapt, build upon this work noncommercially, and license their derivative works on different terms, provided the original work is properly cited and the use is non-commercial. See: http:// creativecommons.org/licenses/by-nc/4.0/

\section{REFERENCES}

1. Heidari B. Knee osteoarthritis prevalence, risk factors, pathogenesis and features: part I. Caspian J Intern Med 2011;2:205-12.

2. Sharma L, Cahue S, Song J, et al. Physical functioning over three years in knee osteoarthritis: role of psychosocial, local mechanical, and neuromuscular factors. Arthritis Rheum 2003;48:3359-70.

3. Dieppe P, Cushnaghan J, Tucker M, et al. The Bristol 'OA500 study': progression and impact of the disease after 8 years. Osteoarthritis Cartilage 2000;8:63-8.

4. Fransen M, McConnell S. Exercise for osteoarthritis of the knee. Cochrane Database Syst Rev 2008;(4):Cd004376.

5. Hochberg MC, Altman RD, April KT, et al. American College of Rheumatology 2012 recommendations for the use of nonpharmacologic and pharmacologic therapies in osteoarthritis of the hand, hip, and knee. Arthritis Care Res (Hoboken) 2012;64:465-74.

6. McAlindon TE, Bannuru RR, Sullivan MC, et al. OARSI guidelines for the non-surgical management of knee osteoarthritis. Osteoarthritis Cartilage 2014;22:363-88.

7. Fransen M, McConnell S, Harmer AR, et al. Exercise for osteoarthritis of the knee. Cochrane Database Syst Rev 2015;1:Cd004376.

8. Zhang W, Nuki G, Moskowitz RW, et al. OARSI recommendations for the management of hip and knee osteoarthritis: part III: changes in evidence following systematic cumulative update of research published through January 2009. Osteoarthritis Cartilage 2010;18:476-99.

9. Bradley LA, Kersh BC, DeBerry JJ, et al. Lessons from fibromyalgia: abnormal pain sensitivity in knee osteoarthritis. Novartis Found Symp 2004:260:258-70; discussion 70-9.

10. Finan PH, Buenaver LF, Bounds SC, et al. Discordance between pain and radiographic severity in knee osteoarthritis: findings from quantitative sensory testing of central sensitization. Arthritis Rheum 2013;65:363-72.

11. Woolf CJ. Central sensitization: implications for the diagnosis and treatment of pain. Pain 2011;152(3 Suppl):S2-15.

12. Lluch E, Torres R, Nijs J, et al. Evidence for central sensitization in patients with osteoarthritis pain: a systematic literature review. Eur J Pain 2014;18:1367-75.

13. Miranda PC, Lomarev M, Hallett M. Modeling the current distribution during transcranial direct current stimulation. Clin Neurophysiol 2006;117:1623-9.

14. Wagner T, Valero-Cabre A, Pascual-Leone A. Noninvasive human brain stimulation. Annu Rev Biomed Eng 2007;9:527-65.

15. Lang N, Siebner HR, Ward NS, et al. How does transcranial DC stimulation of the primary motor cortex alter regional neuronal activity in the human brain? Eur J Neurosci 2005;22:495-504.

16. Boggio PS, Amancio EJ, Correa CF, et al. Transcranial DC stimulation coupled with TENS for the treatment of chronic pain a preliminary study. Clin J Pain 2009;25:691-5.

17. Fregni F, Gimenes R, Valle AC, et al. A randomized, sham-controlled, proof of principle study of transcranial direct current stimulation for the treatment of pain in fibromyalgia. Arthritis Rheum 2006;54:3988-98.

18. Hummel F, Celnik P, Giraux P, et al. Effects of non-invasive cortical stimulation on skilled motor function in chronic stroke. Brain 2005;128:490-9.

19. Stagg C, Jayaram G, Pastor D, et al. Polarity and timing dependent effects of transcranial direct current stimulation in explicit motor learning. Neuropsychologia 2011;49:800-4.

20. Nitsche MA, Seeber A, Frommann K, et al. Modulating parameters of excitability during and after transcranial direct current stimulation of the human motor cortex. J Physiol 2005;568:291-303.

21. Zaghi S, Thiele B, Pimentel D, et al. Assessment and treatment of pain with non-invasive cortical stimulation. Restor Neurol Neurosci 2011;29:439-51. 
22. Garcia-Larrea L, Peyron R, Mertens $P$, et al. Electrical stimulation of motor cortex for pain control: a combined PET-scan and electrophysiological study. Pain 1999;83:259-73.

23. Strafella AP, Vanderwerf $Y$, Sadikot AF. Transcranial magnetic stimulation of the human motor cortex influences the neuronal activity of subthalamic nucleus. Eur J Neurosci 2004;20: 2245-9.

24. Hoffman MD, Shepanski MA, Ruble SB, et al. Intensity and duration threshold for aerobic exercise-induced analgesia to pressure pain Arch Phys Med Rehabil 2004;85:1183-7.

25. O'Leary S, Falla D, Hodges PW, et al. Specific therapeutic exercise of the neck induces immediate local hypoalgesia. J Pain 2007:8:832-9.

26. Koltyn KF, Arbogast RW. Perception of pain after resistance exercise. Br J Sports Med 1998;32:20-4.

27. Millan MJ. Descending control of pain. Prog Neurobiol 2002;66:355-474.

28. Schabrun S, Chipchase L. Priming the brain to learn: the future of therapy? Man Ther 2012;17:61-6.

29. Gray R, Sullivan M, Altman DG, et al. Adherence of trials of operative intervention to the CONSORT statement extension for non-pharmacological treatments: a comparative before and after study. Ann R Coll Surg Engl 2012;94:388-94.

30. Hoffmann TC, Glasziou PP, Boutron I, et al. Better reporting of interventions: template for intervention description and replication (TIDieR) checklist and guide. BMJ 2014;348:g1687.

31. Altman R, Asch E, Bloch D, et al. Development of criteria for the classification and reporting of osteoarthritis. Classification of osteoarthritis of the knee. Diagnostic and Therapeutic Criteria Committee of the American Rheumatism Association. Arthritis Rheum 1986;29:1039-49.

32. Gandiga PC, Hummel FC, Cohen LG. Transcranial DC stimulation (tDCS): a tool for double-blind sham-controlled clinical studies in brain stimulation. Clin Neurophysiol 2006;117:845-50.
33. Lange AK, Vanwanseele B, Fiatarone Singh MA. Strength training for treatment of osteoarthritis of the knee: a systematic review. Arthritis Rheum 2008;59:1488-94.

34. Roddy E, Zhang W, Doherty M, et al. Evidence-based recommendations for the role of exercise in the management of osteoarthritis of the hip or knee-the MOVE consensus. Rheumatology (Oxford) 2005;44:67-73.

35. Arendt-Nielsen L, Nie H, Laursen MB, et al. Sensitization in patients with painful knee osteoarthritis. Pain 2010;149:573-81.

36. Wylde V, Palmer S, Learmonth ID, et al. Test-retest reliability of Quantitative Sensory Testing in knee osteoarthritis and healthy participants. Osteoarthritis Cartilage 2011;19:655-8.

37. Lewis GN, Heales L, Rice DA, et al. Reliability of the conditioned pain modulation paradigm to assess endogenous inhibitory pain pathways. Pain Res Manag 2012;17:98-102.

38. Courtney CA, Lewek MD, Witte PO, et al. Heightened flexor withdrawal responses in subjects with knee osteoarthritis. J Pain 2009;10:1242-9.

39. Micalos PS, Drinkwater EJ, Cannon J, et al. Reliability of the nociceptive flexor reflex (RIII) threshold and association with Pain threshold. Eur J Appl Physiol 2009;105:55-62.

40. Carlesso LC, Macdermid JC, Santaguida LP. Standardization of adverse event terminology and reporting in orthopaedic physical therapy: application to the cervical spine. J Orthop Sports Phys Ther 2010;40:455-63.

41. Poreisz C, Boros K, Antal A, et al. Safety aspects of transcranial direct current stimulation concerning healthy subjects and patients. Brain Res Bull 2007;72:208-14.

42. Abbott $\mathrm{JH}$. The distinction between randomized clinical trials (RCTs) and preliminary feasibility and pilot studies: what they are and are not. J Orthop Sports Phys Ther 2014;44:555-8.

43. Ribeiro DC, Sole G, Abbott JH, et al. The effectiveness of a lumbopelvic monitor and feedback device to change postural behavior: a feasibility randomized controlled trial. J Orthop Sports Phys Ther 2014;44:702-11. 